\title{
Selectivity in normal-phase liquid chromatography with binary mobile phase
}

\author{
Małgorzata Borówko • Barbara Ościk-Mendyk
}

Received: 26 February 2010 / Accepted: 13 May 2010 / Published online: 30 June 2010

(C) The Author(s) 2010. This article is published with open access at Springerlink.com

\begin{abstract}
A simple theoretical approach to normal-phase liquid chromatography with binary mobile phase is presented. In the model used the driving force for the retention is competitive adsorption of solutes and both solvents. A new expression for the separation factor is proposed and discussed. An influence of different parameters on the selectivity is shown. The theoretical concepts are illustrated by the selected experimental data.
\end{abstract}

Keywords Retention mechanism - Liquid-solid chromatography $\cdot$ Selectivity $\cdot$ Adsorption from solutions

\section{Introduction}

Currently, liquid chromatography is the main method for separating compounds in solutions. A flow of the mobile phase along the stationary phase causes differentiated migration of solutes in chromatographic bed. The relative preference of the solute molecules for the two phases results in a solute distribution over the mobile and stationary phase. Knowledge of the mechanism of the retention is of the utmost importance for the control of separation and optimization of analysis time. In normal-phase liquid chromatography the driving force for the transfer of solute is its adsorption on the solid surface. It is generally acknowledged that the adsorption depends on parameters characterizing either the solute or the mobile phase and the adsorbent surface. The use mixed mobile phase allows the continuous change of the elution strength of the mobile phase and the

M. Borówko $(\bowtie) \cdot$ B. Ościk-Mendyk

Faculty of Chemistry, M. Curie-Skłodowska University,

M. Curie-Skłodowska Sq. 3, 20-031 Lublin, Poland

e-mail: borowko@hektor.umcs.lublin.pl selectivity of the chromatographic system. Such a manipulation of separation requires the functional relationships between retention factors and the mobile phase composition. Numerous models have been developed to describe the retention in liquid solid chromatography with mixed mobile phases, several reviews cover the results (Borówko 2002; Borówko and Jaroniec 1983a, 1983b; Jaroniec et al. 1985; Nurok 1989; Snyder 1969; Soczewiński 2002). These approaches involve various factors, namely, interactions with the surface (Glajch and Snyder 1981; Jandera et al. 2001; Jaroniec et al. 1978; Scott and Kucera 1975; Snyder and Glajch 1981), non-specific interactions in the liquid phase (Boehm and Martire 1980; Borówko 1984) association and solvation effects (Borówko and Jaroniec 1983a, 1983b; Jaroniec et al. 1985) differences in molecular sizes of components (Borówko 1986)), energetical heterogeneity of the adsorbent (Borówko 2002; Borówko and Jaroniec 1979; Jaroniec and Ościk-Mendyk 1981), orientation of solute molecules on the surface (Borówko 1988), etc. Nevertheless, theoretical aspects of liquid adsorption chromatography still remain a matter of the debate in the literature.

In this paper we focus our attention on separation of solutes which have different molecular sizes and different adsorptive properties. In Sect. 2 the theory is briefly described. A new equation for separation factor is proposed and analyzed. Section 3 gives details connected with the experiments. To measure the retention factors thin layer chromatography has been used. Results are discussed in Sect. 4. We present several theoretical dependences for the separation factor as a function of mobile phase composition. These relationships are illustrated by selected experimental data. We also interpret the experimental results measured for different mixed mobile phases and different adsorbents. 


\section{Theory}

Let us discuss a simple model of retention in the liquid-solid chromatography with a binary mobile phase. We consider the system which consists of a solute 's', a binary mobile phase $(1,2)$ and an energetically homogeneous adsorbent. A monomolecular layer of liquid immediately adjacent to the adsorbent surface is treated as the stationary phase. We assume that molecules of both solvents have the same size. However, molecular sizes of solutes can be different. Solute molecules are adsorbed parallel to the solid surface. Driving force of retention is interaction with the adsorbent. We neglect molecular interactions in the liquid mixture. Numerous studies have shown that the assumptions are quite reasonable for many chromatographic systems and the model describes the most salient features of retention (Borówko and Jaroniec 1983a, 1983b; Borówko and Ościk-Mendyk 2005; Jaroniec et al. 1985). Indeed, the molecular sizes of the majority of solvents used in the normal-phase liquid chromatography are very similar or at least they are of the same order of magnitude. It has been also shown that if interactions with surface are sufficiently strong the non-ideality of a mixture and the heterogeneity of the surface may be neglected.

Adsorption from liquid mixtures has a competitive character, so the process can be described by the following phase-exchange reactions (Jaroniec et al. 1985)

$(i)^{l}+r_{i j}(j)^{\sigma} \Leftrightarrow(i)^{\sigma}+r_{i, j}(j)^{l}$

where the symbol $(j)^{\rho}$ denotes the molecule ' $j$ ' $(j=$ $s, 1,2)$ in the $\rho$-th phase; the superscripts $l$ and $\sigma$ refer to the mobile and stationary phases, respectively. The stoichiometric coefficient $r_{i j}$ is the average number of molecules of the $j$-th component removed from the adsorbent surface by a single molecule ' $i$ '. In the considered case $r_{12}=1$, $r_{s 1}=r_{s 2}=r_{s}$. The parameter $r_{s}$ is equal to the ratio of the partial molar areas of the solute and the solvent. The partial molar area is defined as $a_{i}=\left(\frac{\partial A}{\partial n_{i}^{\sigma}}\right)_{T, P, n_{j \neq i}^{\sigma}}, A$ is the specific area of the surface and $n_{i}^{\sigma}$ denotes the number moles of the $i$-th component in the stationary phase (Everett 1964).

At the equilibrium we may write

$K_{s 1}=\frac{y_{s}}{x_{s}}\left(\frac{x_{1}}{y_{1}}\right)^{r_{s}}$

where $K_{s 1}$ is the thermodynamic constant of the reaction (1) for $i=s$ and $j=1 ; x$ and $y$ denote the mole fractions in the mobile and the stationary phase, respectively. The constant $K_{s 1}$ characterizes adsorption of the solute from the 1st solvent.

The distribution ratio of the solute is equal to

$k_{s}=y_{s} / x_{s}$
The retention factor is proportional to the distribution ratio defined above

$k_{s}^{\prime}=q \cdot k_{s}$

where $q$ is the constant characteristic for the chromatographic bed and independent of the solvent (Snyder 1969).

Combination of (2) and (3) leads to the general equation for the distribution ratio

$k_{s}=K_{s 1} Y^{r_{s}}$

with

$Y=Y\left(x_{1}\right)=y_{1} / x_{1}$

In the chromatographic process the solute concentration is infinitely low $\left(x_{s} \rightarrow \infty\right)$ so the solute does not affect adsorption of the 1 st solvent. As a consequence, the function $Y\left(x_{1}\right)$, that describes the distribution of the polar solvent between the stationary and mobile phases, is independent of the solute. It can be estimated immediately from adsorption measurements (Jaroniec and Ościk-Mendyk 1981) or calculated from the well-known theoretical isotherm proposed by Everett (1964)

$y_{1}=K_{12} x_{1} /\left(K_{12} x_{1}+x_{2}\right)$

where $K_{12}$ is the thermodynamic constant of reaction for $i=1$ and $j=2$.

Taking into account that $x_{2}=1-x_{1}$ we obtain

$Y=\left[a x_{1}+b\right]^{-1}$

for

$a=1-K_{21}, \quad b=K_{21}, \quad K_{21}=1 / K_{12}$

The constant $K_{i j}$ that describes adsorption of the $i$ th component from the binary liquid mixture $(i, j)$ is given by (Borówko 1986; Jaroniec et al. 1985)

$K_{i j}=s_{i j} \exp \left[\left(E_{i}-r_{i j} E_{j}\right) / k_{B} T\right]$

where $E_{i}$ is adsorption energy for a molecule of the $i$ th component, $s_{i j}$ is the entropy factor, $k_{B}$ is the Boltzmann constant. At first approximation we assume that $s_{i j}=1$ $(i, j=s, 1,2)$.

To analyze effects of molecular sizes in liquid adsorption chromatography we introduce the following scaling of energy

$\varepsilon_{i}=E_{i} / r_{i 1}$

A solvent molecule is treated as a unit of molecular size (segment). The parameter $\varepsilon_{i}$ corresponds to adsorption energy of such a segment of molecule ' $i$ '. 
The corresponding Langmuir constant is equal to

$k_{i o}=\exp \left(\varepsilon_{i} / k_{B} T\right)$

In our model the following relationship is satisfied (Borówko 1986)

$K_{12}=\left(k_{s(2)} / k_{s(1)}\right)^{1 / r_{s}}$

where $k_{s(i)}$ is the distribution ratio of the solute ' $s$ ' in a pure solvent ' $i$ '.

The distribution ratio in a mixed mobile phase can be expressed by means of the distribution ratios in pure solvents (Borówko 1986)

$k_{s}=\left[\sum_{i=1}^{2} x_{i} /\left(k_{s(i)}\right)^{1 / r_{s}}\right]^{-r_{s}}$

We assume that the solvent " 1 " is more polar than solvent ' 2 ', so it is preferentially adsorbed from the binary mixture $(1,2)$. This means that $K_{12}>1$ and $a>0$. For such adsorptive properties of the mobile phase the solute distribution ratio calculated from (5) and (8) decreases monotonically with increasing concentration of the polar solvent.

A separation of two sample components, say the solute " $s$ " and the solute " $p$ " is characterized by the separation factor

$\alpha_{s p}=k_{s} / k_{p}$

In the framework of our model the separation factor is given by

$\alpha_{s p}=C_{s p(1)} Y^{\Delta r_{s p}}$

where

$\Delta r_{s p}=r_{s}-r_{p}$

and $C_{s p(1)}$ is a parameter independent of the mobile phase composition

$$
\begin{aligned}
C_{s p(1)} & =\left(k_{s o}\right)^{r_{s}}\left(k_{p o}\right)^{-r_{p}}\left(k_{1 o}\right)^{-\Delta r_{s p}} \\
& =\exp \left[\left(E_{s}-E_{p}-\Delta r_{s p} E_{1}\right) / k_{B} T\right]
\end{aligned}
$$

Now, we discuss conclusions following from (16). If the both solutes have the same sizes $\left(\Delta r_{s p}=0\right)$ the separation factor does not depend on a composition mobile phase

$\alpha_{s p}=K_{s p}=\mathrm{const}$

In this case the solvent strength can be controlled whereas the selectivity remains constant.

When a molecule of the solute " $s$ " is smaller than a molecule of the solute " $p$ " $\left(\Delta r_{s p}<0\right)$ the separation factor $\alpha_{s p}$ is a monotonically increasing function of the mole fraction of a polar solvent. However, for $r_{s}>r_{p}\left(\Delta r_{s p}>0\right)$ the separation factor decreases with an increasing concentration of a polar solvent " 1 ".

One can also consider two interesting special cases of (16). If a molecule of a given solute pushes out from the stationary phase one more solvent molecule than a molecule of the other solute pushes out $\left(\left|\Delta r_{s p}\right|=1\right)$ the separation factor can be a linear function of the mobile phase composition. For $r_{s}<r_{p}$ we have

$\alpha_{s p}=a^{*}+b^{*} x_{1}$

where $a^{*}=C_{s p(1)} a$ and $b^{*}=C_{s p(1)} b$.

However, for $r_{s}>r_{p}\left(\Delta r_{s p}=1\right)$ the separation factor $\alpha_{p s}=\left(\alpha_{s p}\right)^{-1}$ is a linear function of a volume fraction $x_{1}$. In the case of $\left|\Delta r_{s p}\right|=2$ we obtain parabolic dependences $\left(\alpha_{s p} v s x_{1}\right.$ for $r_{s}<r_{p}$ and $\alpha_{p s} v s x_{1}$ for $\left.r_{s}>r_{p}\right)$.

\section{Experimental}

Chromatography was performed on $10 \mathrm{~cm} \times 10 \mathrm{~cm}$ glass plates with silica gel $60 \mathrm{HF}_{254}$ and aminopropyl-, cyanopropyl and diol-polar-bonded stationary phases (Merck, Darmstadt, FRG). Plates were developed, with the adsorbent layer face-down in horizontal, equilibrated DS chambers (Chromdes, Lublin, Poland). Before development the chambers were conditioned by 5 minutes in the vapor of the polar mobile phase component. The mobile phase components were: n-heptane, cyclohexane, carbon tetrachloride, toluene, chloroform, ethylene chloride and tetrahydrofuran. The molar fraction of polar solvent in the mobile phase ranged from 0.2 to 0.8 , depending on the kind of chromatographic system. Chromatographic measurements were made for 10-20 test substances. Several of them were new potential herbicides, $\mathrm{N}$-aryl-trichloroacetamides and 2-(chlorophenoxy)acyl derivatives synthesized in Department of Organic Chemistry and Technology of Faculty of Chemistry, UMCS. Solutions of test substances were prepared in methanol and ethylene chloride. Samples, $1 \mu \mathrm{L}$, were automatically applied on the plates by means of a Camag Linomat IV applicator. Detection was performed by Shimadzu CS-9000 dual-wavelength Scanner at $\lambda=$ $200 \mathrm{~nm}$.

\section{Results and Discussion}

Figures 1 and 2 present results of theoretical calculations performed using (5) for the retention ratio and (16) for the separation factor. We consider solutes belonging to the same 'homologous series', we assume that the adsorption energy of one 'segment' $\left(\varepsilon_{s}\right)$ is fixed. The theory predicts several 

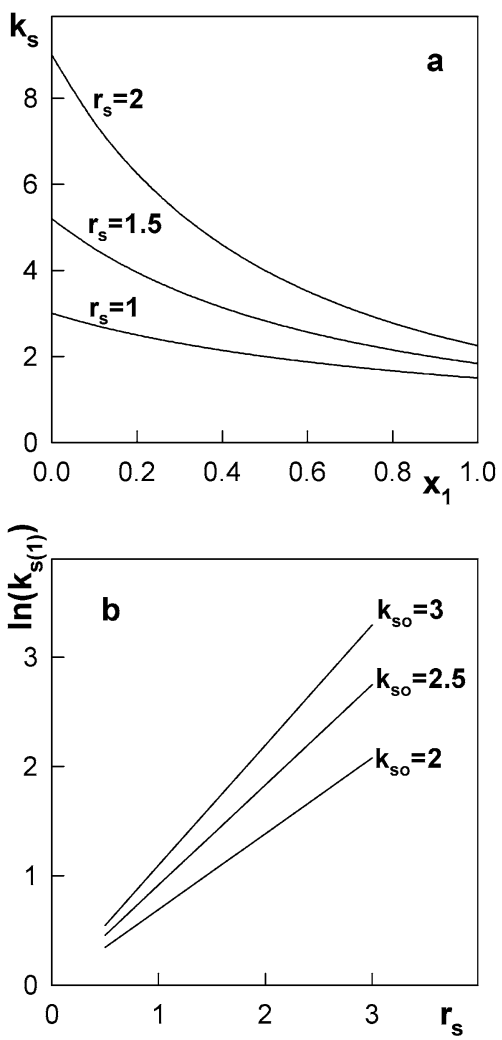

Fig. 1 (a) Theoretical dependences of the retention ratio on the mobile phase composition for solutes of different molecular sizes; (b) An influence of molecular size of the solute and its adsorption energy on the retention factor in a pure solvent ' 1 '. Parameters: $k_{1 o}=2$ (a) $k_{2 o}=1$, $k_{s o}=3, r_{s}=1,1.5,2$; (b) $k_{s o}=2,2.5,3$

important features of retention. First, the retention ratio increases with solute size (Fig. 1a). Larger solutes are retained longer than smaller ones. Second, the logarithm of the retention ratio depends linearly on the size of the solute molecule. The slopes of the straight lines increase with increasing adsorption energy of the solute (increasing $k_{s o}$ ) (Fig. 1b).

Figure 2 demonstrates dependences of separation factors $\alpha_{s p}$ on the volume fraction of a more polar solvent in a mobile phase. In Fig. 2a separation factors for several monomer/r-mer pairs $\left(r_{s}=1, r_{p}>1\right)$ are plotted. In this case the separation factors increase with $x_{1}$. The separation is better in nonpolar solvent. The separation factor for the monomer/dimer sample changes linearly with the mole fraction $x_{1}$. For the monomer/trimer pair one observes a parabolic dependence. Illustrated in Fig. $2 b$ is the effect of mobile phase on the separation factor for the monomer/dimer pair. As the solvent ' 1 ' becomes more polar, its adsorption increases, leading to an increase in the separation factor. The use of a stronger adsorbent also causes an increase in the separation factor.

We have determined retention ratios for many solutes and calculate the separation factors for different pairs of the solutes. Figures 3-5 illustrate an influence of the mo-
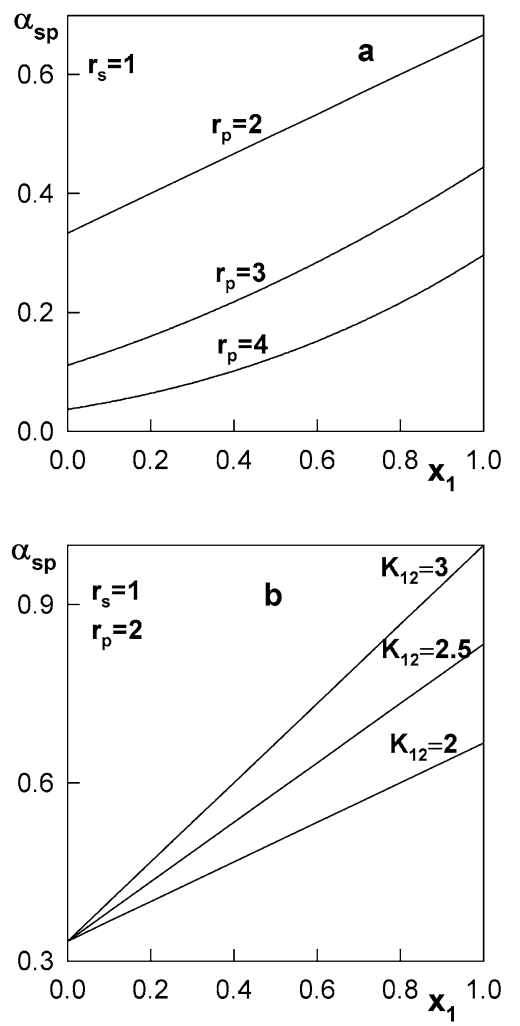

Fig. 2 Theoretical dependences of the separation factor on the mobile phase composition for different pairs of solute: (a) monomer/r-mer; (b) monomer/dimer in different mobile phases. Parameters: $k_{2 o}=1$, $k_{s o}=k_{p o}=3, r_{s}=1$; (a) $k_{1 o}=2$ and $r_{p}=2,3,4$; (b) $r_{p}=2$ and $k_{1 o}=K_{12} \cdot k_{2 o}=2,2.5,3$

bile phase composition on the separation factor for selected chromatographic systems.

We begin with presentation of linear relationships between the separation factor and the mobile phase composition. In the case of solute pairs in which a molecule ' $s$ ' is smaller than a molecule ' $p$ ' (Fig. 3a) one see that when a volume fraction of a polar solvent increases the separation factor $\alpha_{s p}$ increases as well. The slopes depend on differences in molecular sizes. However, if molecular sizes of both solutes are similar the separation factor $\alpha_{s p}$ does not depend on the mobile phase composition (Fig. 3b). These results confirm our theory.

It is interesting to discuss how different parameters affect the separation factor. We analyze an influence of the mobile phase, the adsorbent and the nature of solutes on the selectivity. Figure 4 shows the curves $\alpha_{s p} v s x_{1}$ for trichloroacetanilide $(s)$ and $\mathrm{N}$-(2,4-dichlorophenyl)-trichloroacetamide $(p)$ adsorbed from $n$-heptane (solvent ' 2 ') mixed with chloroform, ethylene chloride or tetrahydrofuran (solvent ' 1 ') on three adsorbents: (a) silica gel, (b) diol-bonded and (c) cyano-bonded. As it is well known, the polarities of solvents increase in the following sequence: chloroform $<$ ethylene chloride $<$ tetrahydrofuran. In this case the use of more polar solvent causes an increase of the separation factor. Com- 

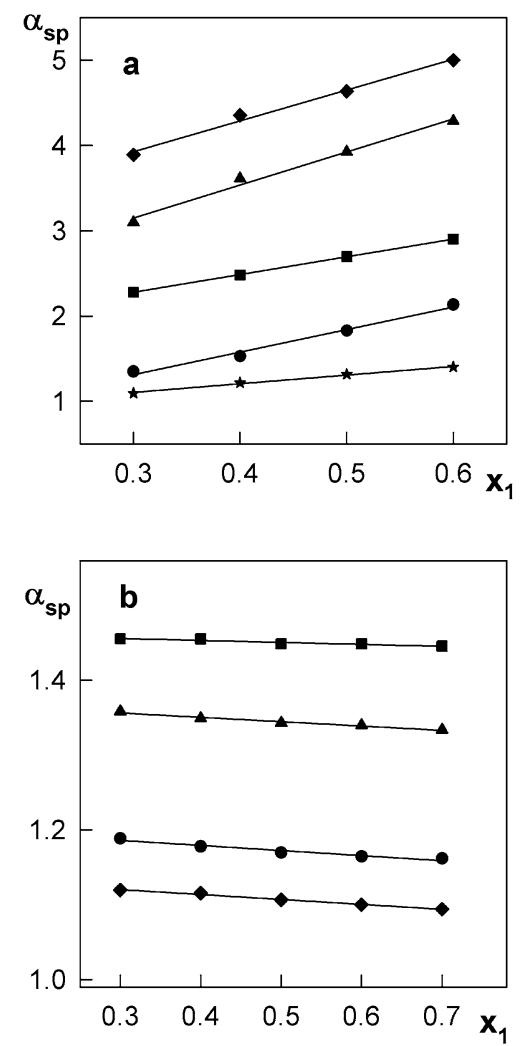

Fig. 3 The dependence of the separation factor $\alpha_{s p}$ on the mole fraction of the polar solvent for (a) the $n$-heptane-ethylene chloride on amino-bonded stationary phase and the following pairs of solutes: 8-hydroxyquinoline/4-amino-3-nitrotoluene (diamonds), 8-hydroxyquinoline/2-nitroaniline (triangles), 8-hydroxyquinoline/4-nitro-2-aminotoluene (squares), 8-hydroxyquinoline/5nitro-2-aminotoluene (circles), acridine/2-nitroaniline (stars), (b) 4-toluidine/2-toluidine in cyclohexane-carbon tetrachloride on silica gel (squares), 3-nitroaniline/4-nitro-2-aminotoluene in $n$-heptane-ethylene chloride o aminopropyl polar-bonded phase (triangles), N-(3-chlorophenyl)-trichloroacetamide/trichloroacetanilide in $n$-heptane-chloroform on diol-bonded silica (circles), 2.3-dichlorophenol/2.5-dichlorophenol in toluene-chloroform on silica gel (diamonds)

paring the corresponding curves in the parts (a), (b) and (c) one can conclude that a type of the surface affect separation factor in the predicted way.

In Fig. 5 we present the results obtained for the pair of solutes different from that discussed above: N-(2-chlorophenyl)-trichloroacetamide) $(s)$ and $\mathrm{N}$-(4-methylphenyl)trichloroacetamide $(p)$. Mixed mobile phases and adsorbents are the same as in Fig. 4. When chloroform and ethylene chloride are used as polar modifiers the separation factors calculated for the same mobile phase and different adsorbents satisfy the relation $\alpha_{s p}\left(\mathrm{SiO}_{2}\right)<\alpha_{s p}$ (Diol) $<\alpha_{s p}(\mathrm{CN})$. However, for the mobile phases containing tetrahydrofuran the sequence changes: $\alpha_{s p}(\mathrm{CN})<\alpha_{s p}(\mathrm{Diol})$ $<\alpha_{s p}\left(\mathrm{SiO}_{2}\right)$. The retention mechanism in such systems is likely more complicated. The mobile phase is non-ideal and it can affect the selectivity of adsorption. A comparison of
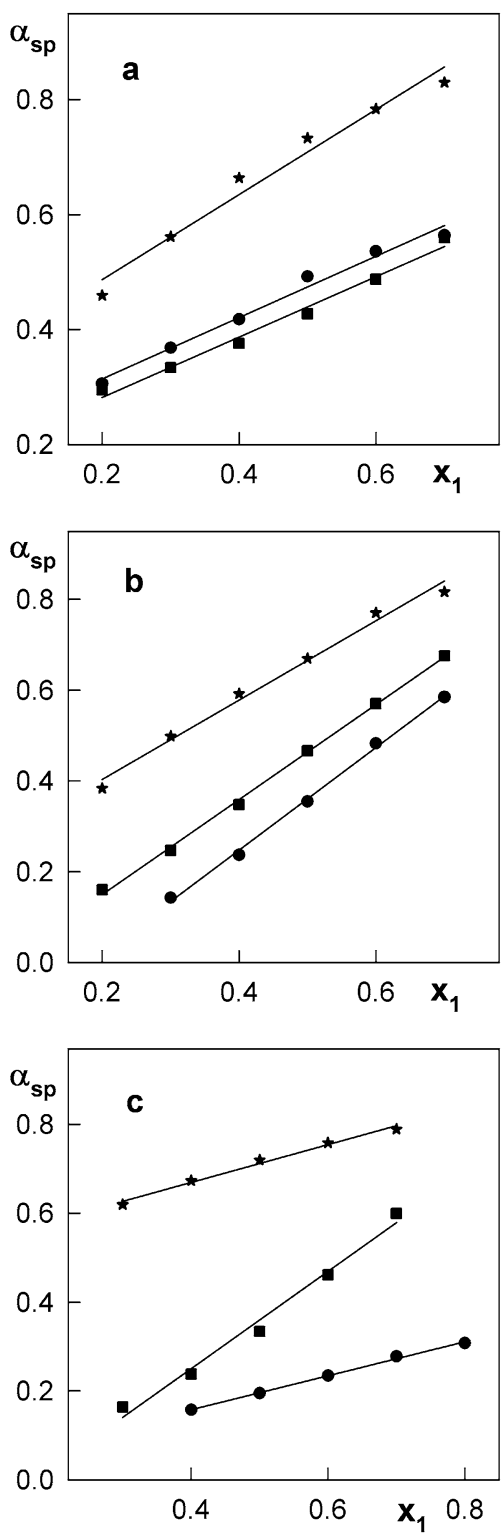

Fig. 4 The dependence of the separation factor $\alpha_{s p}$ on the mole fraction of the polar solvent for the solute pair: trichloroacetanilide $(s)$ and $\mathrm{N}$-(2,4-dichlorofenyl)-trichloroacetamide adsorbed from $n$-heptane mixed with chloroform (circles), ethyl chloride (squares), tetrahydrofuran (stars) on (a) cyano-bonded, (b) diol-bonded stationary phases and (c) silica gel

Figs. 4 and 5 proves that selectivity strongly depends on a nature of both solutes.

\section{Conclusions}

The retention and selectivity in normal-phase liquid chromatography is strongly affected by molecular sizes of the sample components. This is a simple consequence of the competitive mechanism of adsorption of solutes and solvents on the solid surface. The presented theoretical ap- 

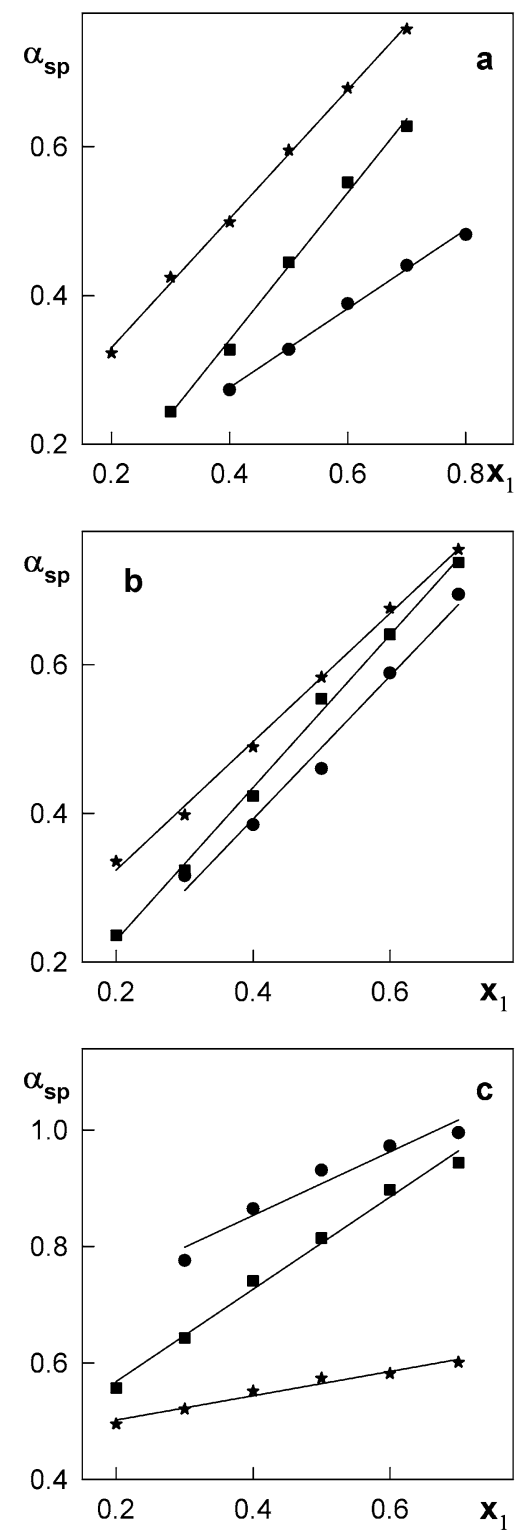

Fig. 5 The dependence of the separation factor $\alpha_{s p}$ on the mole fraction of the polar solvent for the solute pair: $\mathrm{N}$-(2-chlorophenyl)-trichloroacetamide $(s)$ and $\mathrm{N}$-(4-methylphenyl)-trichloroacetamide $(p)$ adsorbed on cyano-bonded (stars), diol-bonded (squares) stationary phases and silica gel (circles) from $n$-heptane mixed with (a) chloroform, (b) ethylene chloride and (c) tetrahydrofuran (solvent ' 1 ')

proach leads to the simple relationship between the selectivity and composition of the mobile phase. The separation factor of a given pair of solutes depends on the thermodynamic constant characterizing adsorption from binary solvent mixture, the difference in molecular sizes of solutes, the ratio of retention factors in the polar solvent and the mobile phase composition. However, as purely adsorption mechanism of retention is assumed the separation factor of solutes having the same molecular sizes is independent of the mobile phase composition. In this case retention of the solutes decreases with increasing the mole fraction of polar solvent but the separation factor remains fixed. For a certain relation between the molecular sizes of solutes the separation factor linearly varies with the mobile phase composition. These theoretical predictions have been confirmed by the comparison with the experimental data.

It must be realized that each model is an incomplete description of a more complex reality. For instance, the surface of the adsorbent is often heterogeneous. Similarly, the solute molecules can be differently oriented to the surface, as is in the case of long linear chains. Furthermore, the molecular interactions in the liquid phase usually play a significant role in the retention process. All these complications are not accounted for in the proposed theory. Nevertheless, our approach gives a new insight into the adsorption mechanism and it can be easily used to optimize the separation in solid liquid chromatography.

Open Access This article is distributed under the terms of the Creative Commons Attribution Noncommercial License which permits any noncommercial use, distribution, and reproduction in any medium, provided the original author(s) and source are credited.

\section{References}

Boehm, R.E., Martire, D.E.: A unified theory of retention and selectivity in liquid chromatography. 1. Liquid-solid (adsorption) chromatography. J. Phys. Chem. 84, 3620-3630 (1980)

Borówko, M.: Liquid adsorption chromatography with multicomponent mobile phase. J. Colloid Interface Sci. 102, 519-526 (1984)

Borówko, M.: A simple dependence of the capacity ratio on the composition of the mobile phase in liquid-solid chromatography. J. Liq. Chromatogr. 9, 1837-1847 (1986)

Borówko, M.: Solute orientation effects in adsorption liquid chromatography. J. Chem. Soc. Faraday Trans. 1 84, 1961-1971 (1988)

Borówko, M.: Adsorption on heterogeneous surfaces. In: Tóth, J. (ed.) Adsorption: Theory, Modeling and Analysis. Surfactant Science Series, vol. 107, pp. 105-173. Dekker, New York (2002)

Borówko, M., Jaroniec, M.: Dependence of the capacity ratio on mobile phase composition in liquid adsorption chromatography. II. Analytical equations for the distribution coefficient involving nonideality of the mobile phase and heterogeneity of the adsorbent surface. Chromatographia 12, 672-678 (1979)

Borówko, M., Jaroniec, M.: Current state in adsorption from multicomponent solutions of nonelectrolytes on solids. Adv. Colloid Interface Sci. 19, 137-177 (1983a)

Borówko, M., Jaroniec, M.: Association effects in adsorption from multicomponent solutions on solids and liquid adsorption chromatography. J. Chem. Soc. Faraday Trans. 1 79, 363-372 (1983b)

Borówko, M., Ościk-Mendyk, B.: Adsorption model for retention in normal-phase liquid chromatography with ternary mobile phases. Adv. Colloid Interface Sci. 118, 113-124 (2005)

Everett, D.H.: Thermodynamics of adsorption from solution. Part 1.Perfect systems. Trans Faraday Soc. 60, 1803-1813 (1964)

Glajch, J.L., Snyder, L.R.: Solvent strength of multicomponent mobile phases in liquid-solid chromatography: Mixtures of three or more solvents. J. Chromatogr. A 214, 21-34 (1981)

Jandera, P., Skavrada, M., Andel, L., Komers, D., Guiochon, G.: Description of adsorption equilibria in liquid chromatography systems with binary mobile phases. J. Chromatogr. A 908, 3-17 (2001) 
Jaroniec, M., Narkiewicz, J., Borówko, M.: Dependence of capacity ratio on mobile phase composition in liquid adsorption chromatography. Chromatographia 11, 581-585 (1978)

Jaroniec, M., Ościk-Mendyk, B.: Application of excess adsorption data measured for components of the mobile phase for characterizing chromatographic systems. J. Chem. Soc. Faraday Trans. 1 77, 1277-1284 (1981)

Jaroniec, M., Martire, D.E., Borówko, M.: Theoretical basis of liquid adsorption chromatography with mixed mobile phases and its connection with the theory of adsorption from multicomponent solutions. Adv. Colloid Interface Sci. 22, 177-227 (1985)

Nurok, D.: Strategies for optimizing the mobile phase in planar chromatography. Chem. Rev. 89, 363-375 (1989)
Scott, R.P., Kucera, P.: Solute interactions with the mobile and stationary phases in liquid-solid chromatography. J. Chromatogr. A 112, 425-442 (1975)

Snyder, L.R: Principles of Adsorption Chromatography. Dekker, New York (1969)

Snyder, L.R, Glajch, J.L.: Solvent strength of multicomponent mobile phases in liquid—solid chromatography: Binary-solvent mixtures and solvent localization. J. Chromatogr. A 214, 1-19 (1981)

Soczewiński, E.: Mechanistic molecular model of liquid-solid chromatography: Retention-eluent composition relationships. J. Chromatogr. A 965, 109-116 (2002) 